\title{
Keratosis follicularis spinulosa decalvans: a family study of seven male cases and six female carriers
}

\author{
Loes D M van Osch, Arnold P Oranje, Frank M Keukens, Pieter C van Voorst \\ Vader, Eddy Veldman
}

\begin{abstract}
Keratosis follicularis spinulosa decalvans (KFSD) is a rare $X$ linked disease which is characterised by follicular hyperkeratosis of the skin and corneal dystrophy. Seven male patients and six female carriers are described. Special attention has been paid to the dermatological and ophthalmic markers of KFSD in patients and carriers. The most prominent features present in the male patients were follicular hyperkeratosis, hyperkeratosis of the calcaneal regions of the soles, scarring alopecia of the scalp, absence of eyebrows and eyelashes, and corneal dystrophy accompanied by photophobia. They also had high cuticles on the fingernails which has not been described before. Carriers often have dry skin, minimal follicular hyperkeratosis, and mild hyperkeratosis of the calcaneal areas of the soles. Mild corneal dystrophy without photophobia was observed in one female carrier.
\end{abstract}

Keratosis follicularis spinulosa decalvans (KFSD) is a rare $\mathrm{X}$ linked disease which affects both the skin and the eyes. Since the first description by Siemens ${ }^{1}$ in 1926 , only a few cases have been reported. KFSD is characterised by follicular hyperkeratosis of the skin and corneal dystrophy. The follicular papules are associated with loss of hair, especially on the scalp, eyebrows, and eyelashes. As a result of the corneal dystrophy many patients show marked photophobia. Symptoms are not present at birth, but usually develop in early childhood. Inheritance appears to be $\mathrm{X}$ linked. Female carriers tend to have milder forms without ocular symptoms and signs of KFSD may not be present at all.

In this article we describe 13 additional cases (seven male patients and six female carriers) who are descendants of the family of Siemens. ${ }^{1}$

\section{Case reports}

CASE 1

A male patient (VII.9, fig 1) presented to the Sophia Children's Hospital in Rotterdam at the age of 8 because of a dry, scaling skin and photophobia. According to the history, these symptoms had been present since early infancy. Sweating was reported to be normal.
On dermatological examination, follicular hyperkeratosis was observed on the legs, the extensor side of the arms, and the face. The perinasal area was spared (fig 2A). A more pronounced, partly follicular, hyperkeratosis was observed on his elbows and knees (fig 2B). The skin of the cheeks and the buttocks was extremely dry and cracked. His eyebrows were almost totally absent and showed erythema and follicular plugging (fig 3). The lower eyelashes were sparse but the scalp hair was normal. The fingernails showed some nonspecific dystrophy and high cuticles (fig 4). Some of the teeth showed longitudinal ridging.

Histopathological examination of a follicular hyperkeratotic lesion showed an epidermis with focal parakeratosis and slight follicular hyperkeratosis. Dermal alterations consisted of perifollicular fibrosis and slight perivascular lymphocyte infiltrate.

Ophthalmic examination indicated normal visual acuity $(1 \cdot 0)$ and marked photophobia. The Meibomian glands were visible through the palpebral conjunctiva. The entire cornea showed punctate subepithelial opacities, which did not stain with fluorescein (fig 5), indicating that the superficial layer of the cornea is intact.

\section{CASE 2}

A male patient (VI.28), aged 21, was referred by the plastic surgeon at the University Hospital Groningen. Cutaneous papules developed a few months after birth on the trunk, extremities, and in the eyebrows. Photophobia was present from the age of 9 months, but was temporarily absent from 10 to 19 years. Sweating was normal. Hair growth was poor everywhere, even after puberty, except on the scalp. A reconstructive operation of the eyebrows a year earlier because of hypotrichosis had been unsuccessful. Before performing punch grafting a dermatologist (PCvVV) was consulted.

On dermatological examination follicular erythematous hyperkeratosis was observed on the trunk and extremities. Both the eyelashes and the scalp hair were normal. The neck showed some follicular papules and minimal scarring alopecia. The eyebrows were sparse and showed postoperative scarring and erythema. Axillary and pubic hair were almost totally absent. The fingernails showed high cuticles. Teeth were normal. 


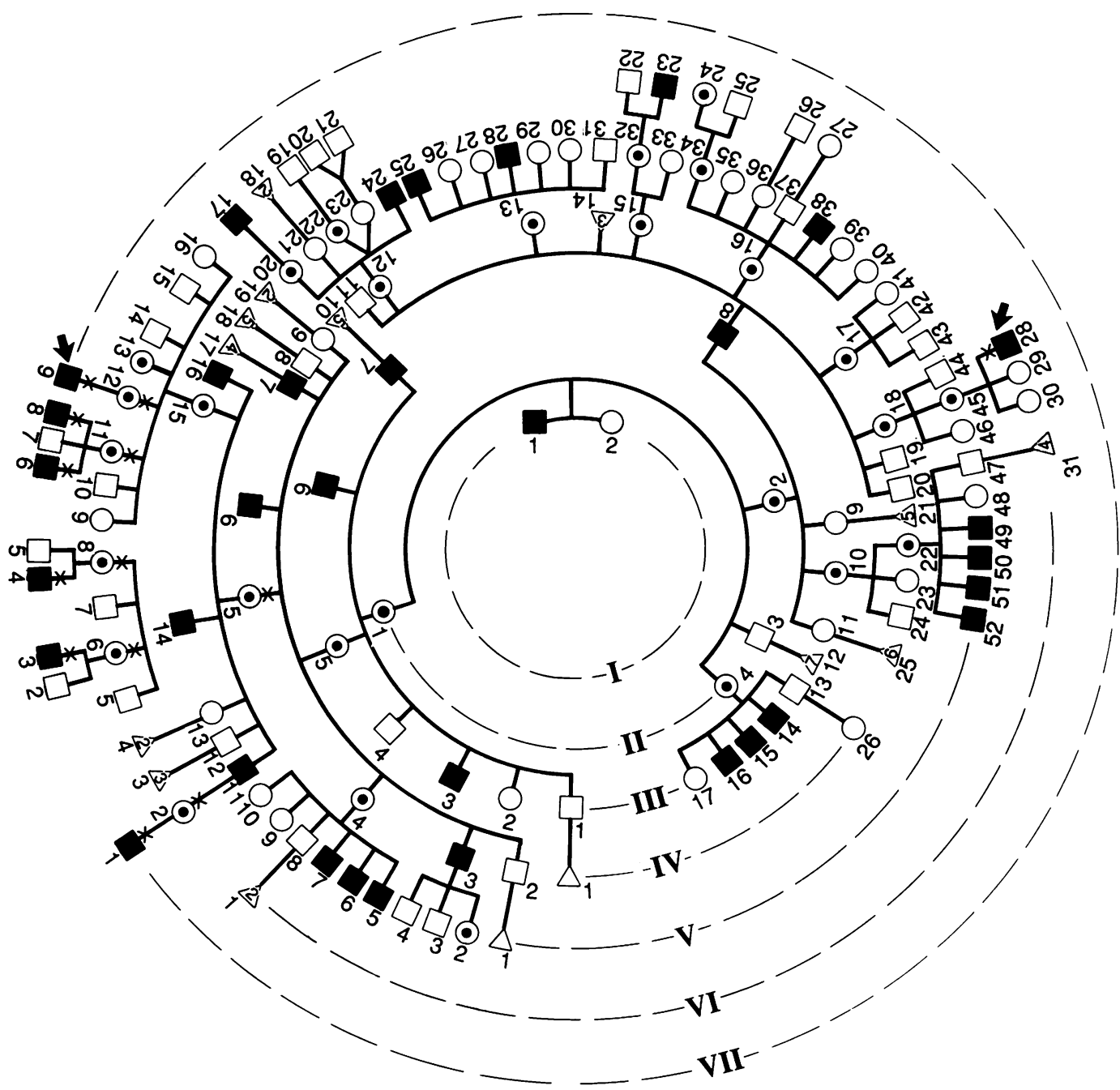

Figure 1 Pedigree of the family with $X$ linked KFSD. Extension of the pedigree published by Waardenburg et al ${ }^{2}$ in 1961. $\odot=$ obligate carrier. $\mathrm{X}=$ examined by authors. Filled squares $=$ affected males. Probands are arrowed.

Biopsy specimens were taken from follicular papules on the neck and trunk. They showed a hair follicle with parakeratosis, hyperkeratosis, and a thickened granular cell layer. The hair shaft was hypoplastic. Sebaceous glands were absent.

Ophthalmic examination indicated normal visual acuity and moderate photophobia. Diffusely spread subepithelial punctate opacities in the cornea were present in large numbers. There were no further abnormalities.

\section{FAMILY EXAMINATION}

According to the history of both patients, other family members were also affected. Genealogical investigation showed that they were both descendants of the family first described by Siemens ${ }^{1}$ in 1926 and later described by Jonkers ${ }^{3}$ in 1950 . Eleven additional family members were examined (five males and six females, fig 1). Clinical data of these family members and both probands are summarised in the table. As indicated in the table, four women had mild follicular hyperkeratosis located on either the knees or the buttocks, while all the males showed severe follicular hyperkeratosis on the face, trunk, and extremities. Prominent follicular hyperkeratosis on the knees was only present in one male (case 1). All affected family members had flesh coloured follicular papules except for case 2 who had papules with erythema. Only one female (VI.6) had some alopecia of the eyebrows and eyelashes while no male patient had normal eyebrows. Three males also had marked scarring alopecia on the scalp (fig 2C) and two males had marked absence of eyelashes. Moderate or severe photophobia was noted in all males but in none of the females. Only one female carrier had very mild corneal dystrophy. New associated findings observed in some males and females were mild hyperkeratosis on the calcaneal region of the soles and some dental abnormalities (longitudinal ridging and poor general condition). Five male patients showed the unusual clinical manifestation of high cuticles of the fingernails (fig 4).

\section{Discussion}

Since our male patients belonging to the 'Siemens' family fulfil all the clinical criteria of 
(A)
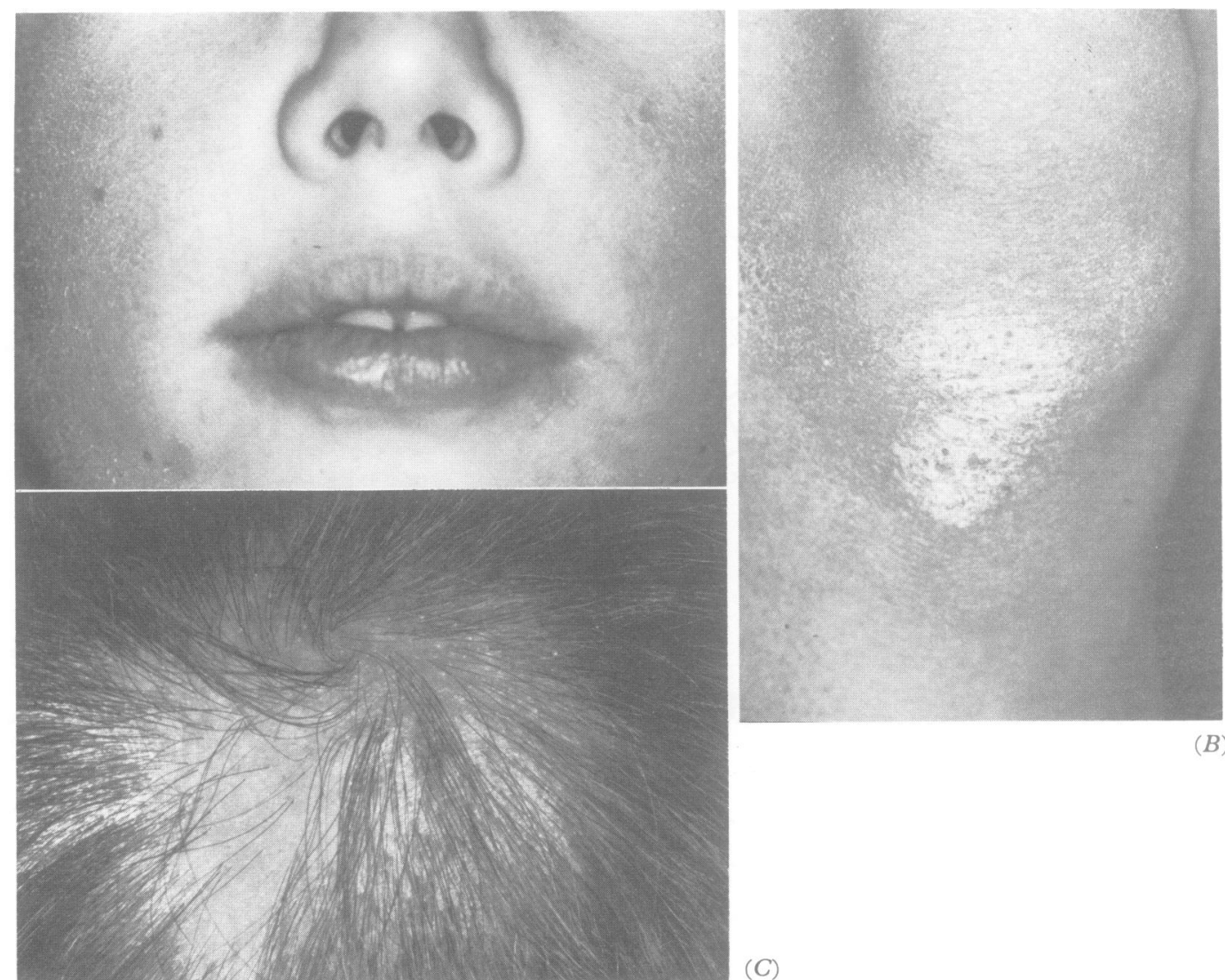

Figure 2 (A) Case 1 (VII.9) showing follicular hyperkeratosis on the face. The perinasal area is spared. (B) Pronounced follicular hyperkeratosis on the knees of case 1. (C) Scarring alopecia on the scalp of patient VII.4.

keratosis follicularis spinulosa decalvans, the diagnosis of KFSD is appropriate. Prominent features of this disease are follicular hyperkeratosis, hyperkeratosis of the calcaneal regions of the soles, scarring alopecia of the scalp, absence of eyebrows and eyelashes, and corneal dystrophy accompanied by photophobia.

The differential diagnosis includes pityriasis rubra pilaris (PRP) and, especially, ichthyosis follicularis. PRP, of which several types exist, is usually not familial and is

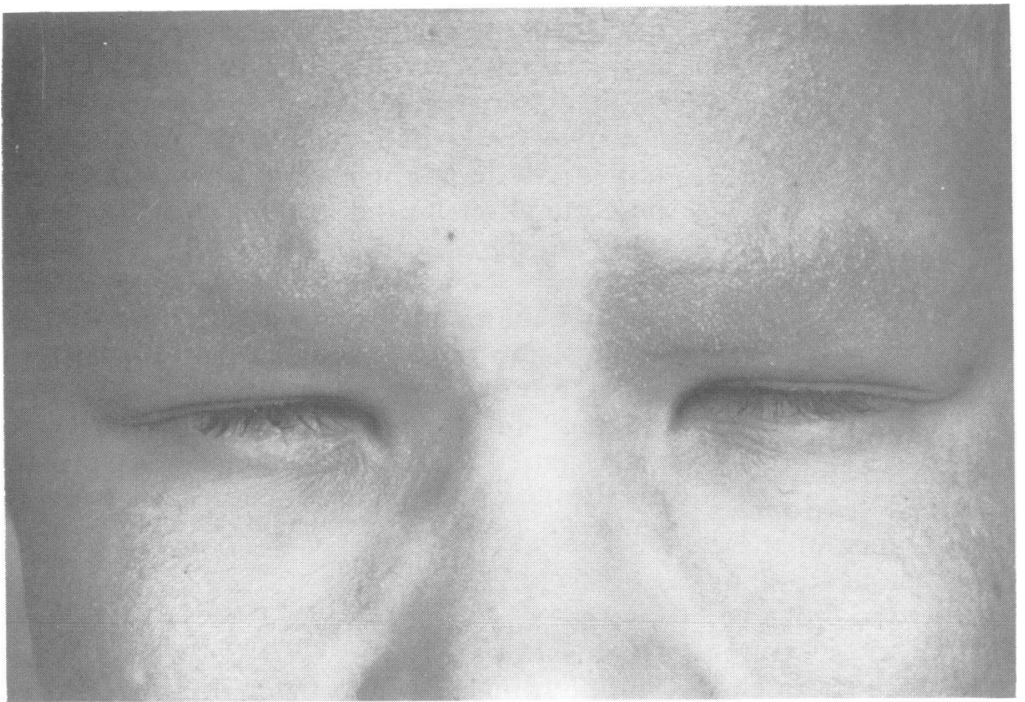

Figure 3 Absence of eyebrows with erythema and follicular plugging in case 1. characterised by a diffuse palmoplantar keratoderma. In addition, the hair itself is unaffected. Confusion may exist especially with Griffiths's type 5 atypical juvenile PRP. ${ }^{4}$ This variant is mostly familial and is considered by some authors as atypical ichthyosis follicularis.

A clear distinction between ichthyosis follicularis and KFSD is more difficult. It is possible that these entities represent one and the same disorder. According to different reports more extensive alopecia, no follicular scarring, and different histological findings from KFSD are observed in ichthyosis follicularis. ${ }^{56}$ In ichthyosis follicularis normal

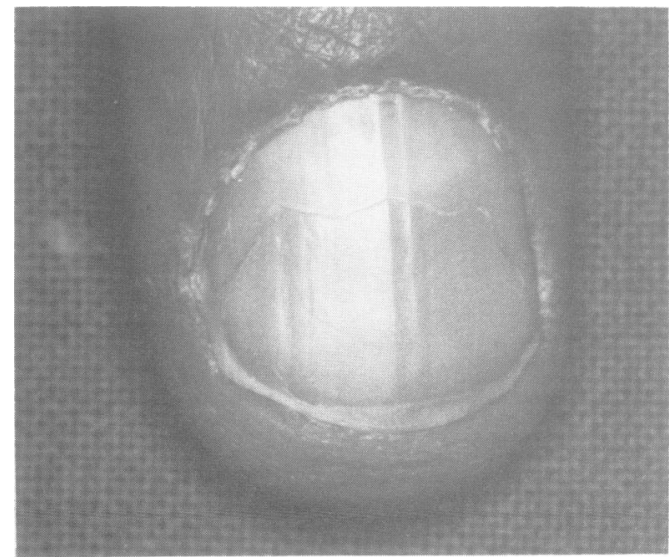

Figure 4 High cuticle of the fingernail in case 1 (VII.9). 
Clinical data of family members.

\begin{tabular}{|c|c|c|c|c|c|c|c|c|c|c|c|c|c|}
\hline & $V \cdot 15$ & $\mathrm{VI} \cdot 2$ & VI 6 & VI 8 & $\mathrm{VI} \cdot 11$ & $\mathrm{VI} \cdot 12$ & VII 1 & VII 3 & VII 4 & VII.6 & VII $\cdot 8$ & VII.9 & $\mathrm{VI} \cdot 28$ \\
\hline Sex & $\mathbf{F}$ & $\mathbf{F}$ & $\mathbf{F}$ & $\mathbf{F}$ & $\mathbf{F}$ & $\mathbf{F}$ & $\mathbf{M}$ & $\mathbf{M}$ & $\mathbf{M}$ & $\mathbf{M}$ & $\mathbf{M}$ & $\mathbf{M}$ & $\mathbf{M}$ \\
\hline Age & 63 & 25 & 35 & 27 & 37 & 35 & 2 & 9 & 5 & 12 & 5 & 8 & 21 \\
\hline Follicular hyperkeratosis & - & - & \pm & \pm & \pm & \pm & ++ & ++ & ++ & ++ & ++ & ++ & ++ \\
\hline Dry skin & - & - & + & + & + & + & ++ & ++ & ++ & ++ & ++ & ++ & ++ \\
\hline Hyperkeratotic heels & - & - & \pm & + & + & + & \pm & - & - & + & - & + & - \\
\hline High cuticles on fingernails & - & - & - & - & - & - & - & + & - & + & + & + & + \\
\hline $\begin{array}{l}\text { Alopecia } \\
\text { Scalp }\end{array}$ & - & - & - & - & - & _ & - & + & + & - & + & - & _- \\
\hline Occiput & - & - & - & - & - & - & + & + & $?$ & - & - & - & + \\
\hline Eyebrows & - & - & + & - & - & - & ++ & ++ & ++ & $+t$ & ++ & ++ & ++ \\
\hline Eyelashes & - & - & \pm & - & - & - & \pm & \pm & + & - & + & \pm & - \\
\hline Teeth & & & & & & & & & & & & & \\
\hline Longitudinal ridges & * & - & * & + & + & - & + & + & - & - & + & + & - \\
\hline Bad condition & * & - & * & + & + & + & - & + & ++ & - & + & + & - \\
\hline Photophobia & - & - & - & - & - & - & ++ & ++ & ++ & + & + & + & + \\
\hline Corneal dystrophy & - & - & - & - & \pm & - & NP & + & + & + & + & + & + \\
\hline Meibomian glands & - & - & - & - & - & - & - & - & + & + & + & + & ? \\
\hline
\end{tabular}

sweat glands, but atrophic or absent sebaceous glands, are found, while in KFSD both glands have been reported as normal. Moreover, patients with KFSD should always have alopecia of the scalp and palmoplantar hyperkeratosis. However, alopecia of the scalp and palmoplantar hyperkeratosis were absent in several of our patients, and in case 2 sebaceous glands were absent in the biopsy specimens. These discordant findings in our patients underline the controversy and confusion in published reports.

We cannot explain why some patients suffer from alopecia. A possible mechanism could be damage to the hair follicles by secondary keratinisation and inflammation. ${ }^{7}$

An interesting new finding in this family is high cuticles on the fingernails of nearly every male patient. This observation has not been previously reported in KFSD. Less specific findings included mild hyperkeratosis on the calcaneal regions of the soles in three males and four females and dental abnormalities in four males and two females.

The photophobia in our male patients can be explained by widely scattered subepithelial opacities, located in the membrane of Bowman and resulting in abnormal refraction of light. Reports concerning the nature of the corneal dystrophy have been controversial. Some

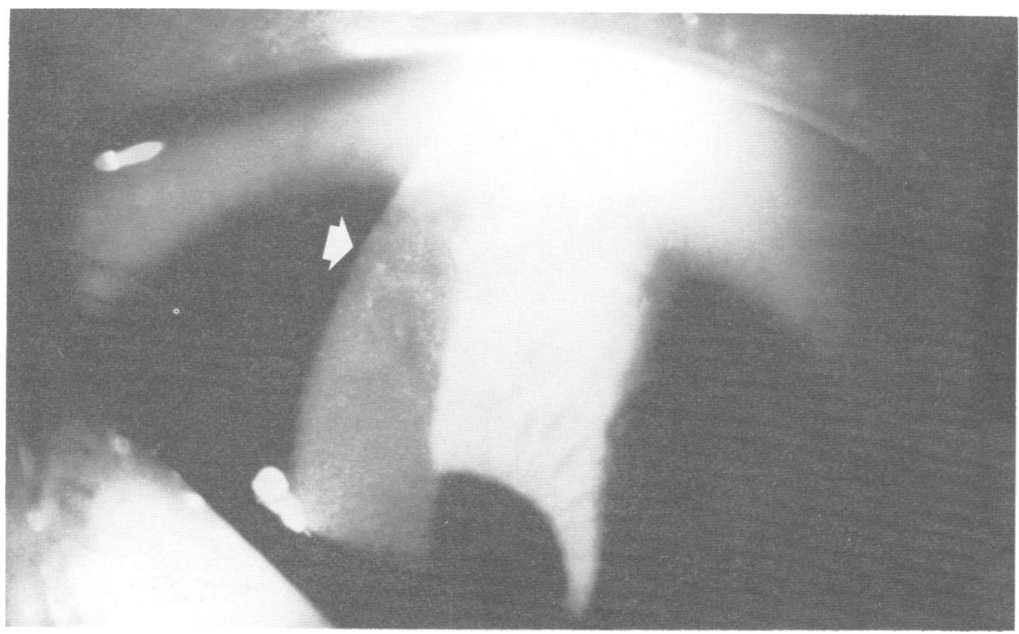

Figure 5 Cornea with punctate subepithelial opacities in case 1 (VII.9). authors consider the opacities to arise from irritation of a more viscous product of the Meibomian glands resulting in formation of hard prickles. In other reports the corneal dystrophy was considered primary as was confirmed by a corneal biopsy. ${ }^{8}$ As we found no evidence of superficial corneal lesions resulting from prickle formation we agree with the latter opinion. According to published reports and the history of our patients both the skin lesions and the photophobia tend to become less severe in puberty.

The pedigree of our patients confirmed the $\mathrm{X}$ linked recessive mode of inheritance. Autosomal dominant inheritance was suggested by Thelen in 1940, indicating genetic heterogeneity. ${ }^{9}$ However, this is incorrect because this family is part of the one originally described by Siemens. Carrier detection of KFSD by clinical examination is very difficult and sometimes impossible. It should be borne in mind, however, that KFSD is a relatively mild disease so carrier detection is of minor importance. As shown in the table, the six female carriers showed no ocular symptoms and skin manifestations were either absent or very mild and not very specific. Female carriers with marked dermatological and ophthalmic symptoms have been reported, however. ${ }^{79}$ It is possible that in the future DNA linkage studies may be helpful in allowing definite genetic differentiation of KFSD, ichthyosis follicularis, and allied diseases.

Retinoids, although theoretically possibly worthwhile, ${ }^{6}$ have never been applied in KFSD. This treatment was proposed in the index cases but was refused because of fear of side effects, especially alopecia. The corneal dystrophy generally requires no specific treatment. Low doses of vitamin A (10000 IU daily) subjectively improved the photophobia of case 2 .

The authors are indebted to B Tank (Department of Dermato-Venereology, University of Rotterdam) for help with the English.

1 Siemens HW. Keratosis follicularis spinulosa decalvans. Arch Dermatol Syphilol 1926;151:384-7.

2 Waardenburg PJ, Franceschetti A, Klein D. Genetics and ophthalmology. Assen: Royal van Gorcum, 1961:520-1.

3 Jonkers GH. Hyperkeratosis follicularis en cornea degenera tie. Ned Tijdschr Geneeskd 1950;51:1464-7.

4 Gelmetti C, Schiuma AA, Cerri D, Gianotti F. Pityriasis rubra pilaris in childhood: a long-term study of 29 cases. Pediatr Dermatol 1986;6:446-51. 
5 Eramo LR, Esterly NB, Zieserl EJ, Stock EL, Herrmann J. Ichthyosis follicularis with alopecia and photophobia. Arch Dermatol 1985;121:1167-74.

6 Hazell M, Marks R. Follicular ichthyosis. Br f Dermatol 1984;111:101-9.

7 Rand R, Baden HP. Keratosis follicularis spinulosa decalvans. Report of two cases and literature review. Arch Dermatol 1983;119:22-6.
8 Franceschetti A, Jaccottet M, Jadassohn W. Manifestations corneennes dans la keratosis follicularis spinulosa decal-

9 Kuokkanen K. Keratosis follicularis spinulosa decalvans in a family from northern Finland. Acta Dermatol Venereol 1971;51:146-50. 